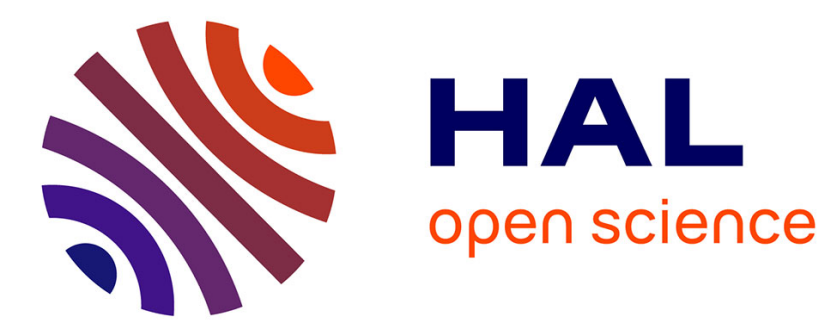

\title{
Cost Models for View Materialization in the Cloud
} Thi-Van-Anh Nguyen, Laurent d'Orazio, Sandro Bimonte, Jérôme Darmont

\section{- To cite this version:}

Thi-Van-Anh Nguyen, Laurent d'Orazio, Sandro Bimonte, Jérôme Darmont. Cost Models for View Materialization in the Cloud. Workshop on Data Analytics in the Cloud (EDBTICDT/DanaC 2012), Mar 2012, Berlin, Germany. http://www.edbt.org/Proceedings/2012Berlin/papers/workshops/danac2012/a2-nguyen.pdf. hal-00686027v2

\section{HAL Id: hal-00686027 \\ https://hal.science/hal-00686027v2}

Submitted on 18 Apr 2012

HAL is a multi-disciplinary open access archive for the deposit and dissemination of scientific research documents, whether they are published or not. The documents may come from teaching and research institutions in France or abroad, or from public or private research centers.
L'archive ouverte pluridisciplinaire HAL, est destinée au dépôt et à la diffusion de documents scientifiques de niveau recherche, publiés ou non, émanant des établissements d'enseignement et de recherche français ou étrangers, des laboratoires publics ou privés.

\section{(c)(1)}

Distributed under a Creative Commons Attribution| 4.0 International License 


\section{Cost Models for View Materialization in the Cloud}

\author{
Thi-Van-Anh Nguyen \\ CNRS, UMR 6072, GREYC \\ Université de Caen Basse \\ Normandie, France \\ thi-van- \\ anh.nguyen@unicaen.fr \\ Sandro Bimonte \\ Irstea, UR TSCF \\ Clermont-Ferrand, France \\ sandro.bimonte@irstea.fr
}

\author{
Laurent d'Orazio \\ CNRS, UMR 6158, LIMOS \\ Université Blaise Pascal \\ Clermont-Ferrand, France \\ laurent.dorazio@univ- \\ bpclermont.fr \\ Jérôme Darmont \\ ERIC Lyon 2, Université de \\ Lyon, France \\ jerome.darmont@univ-lyon2.fr
}

\section{INTRODUCTION}

Cloud computing has recently emerged as a new challenging paradigm. Cloud computing may be defined as a payper-use model for enabling on-demand access to reliable and configurable resources that can be quickly provisioned and released with minimal management. Users only pay for the resources they use. They need not set up the infrastructure, nor buy the software. These features make the design of cloud data management systems desirable. Unfortunately, performance in cloud computing usually relies on scalability, using bigger instances (scale-up) or more nodes (scale-out), making the bill more expensive.

On the other hand, performance optimization in databases has been intensively studied for decades. Reusing methods such as indexing, buffering or view materialization in the cloud could help improving performances without increasing costs. For instance, materialized views, which we particularly focus on in this paper, physically store the results of some relevant, frequent queries. One of the main challenges is then to select what views to materialize. Traditionally, the criteria to consider in the selection process mainly include view storage and maintenance costs $[6,8]$.

In cloud computing, storage is virtually infinite, enabling to store all possible materialized views. However, each materialized view implies an extra storage cost. Thus, the performance optimization problem becomes finding the best tradeoff between raw scalability (i.e., increasing resources) and materialized views under budget constraints. To illustrate the complexity of selecting materialized views in the cloud, let us introduce a simple, fictitious example. Let the storage cost be $\$ 0.10$ per GB and per month, and the computing cost be $\$ 0.24$ per hour. A 500 GB dataset is stored in the cloud for a month. Let $Q$, the monthly query workload, process in 50 hours. Then, storage cost is $\$ 50$, computing cost is $\$ 12$, for a total of $\$ 62$. If some materialized views are used, let us assume that workload processing time becomes 40 hours. Thus, computing cost becomes $\$ 9.6$. However, materialized views use up additional storage space, e.g., 50 GB. Thus, storage cost becomes $\$ 55$, for a total cost of $\$ 64.6$. Overall, performance has improved by $20 \%$, but cost has also increased by $4 \%$.

The selection of views to materialize depends on several parameters, such as the workload (query execution time, database size, etc.), the cloud pricing model (CPU, storage,
Permission to make digital or hard copies of all or part of this work for personal or classroom use is granted without fee provided that copies are not made or distributed for profit or commercial advantage and that copies bear this notice and the full citation on the first page. To copy otherwise, to republish, to post on servers or to redistribute to lists, requires prior specific permission and/or a fee.

Copyright 20XX ACM X-XXXXX-XX-X/XX/XX ...\$10.00. 


\begin{tabular}{|c|c|c|c|c|c|c|}
\hline Year & Month & Day & Country & Region & Department & Profit \\
\hline 2000 & 12 & 31 & France & Auvergne & Puy-de-Dôme & $\$ 35.000$ \\
\hline 2000 & 1 & 1 & France & Auvergne & Puy-de-Dôme & $\$ 40.000$ \\
\hline 2000 & 12 & 31 & Italy & Campanie & Naples & $\$ 23.000$ \\
\hline 1999 & 1 & 1 & Italy & Campanie & Naples & $\$ 50.000$ \\
\hline
\end{tabular}

Table 1: Sales dataset excerpt

network, etc.). This choice is a trade-off between response time and cost, and depends on the needs of a particular user. At one end of the spectrum, users under a strong budget constraint may accept long response times, whereas at the other end, users may disregard cost if they need fast response.

In this paper, we address the multi-criteria optimization problem of selecting a set of materialized views while optimizing the global monetary cost of storing and querying a database in the cloud. To achieve this goal, our main contribution is the design of cost models for storing, maintaining and querying data with materialized views over a cloud architecture.

The remainder of this paper is organized as follows. In Section 2, we provide the background information that is used throughout the paper. In Sections 3 and 4, we define cost models for cloud data management and materializing views, respectively. In Section 5, we describe the optimization process that is based on these cost models. In Section 6 , we present an experimental evaluation and the first performance analyses of our models. In Section 7 , we discuss the state of the art and compare it to our approach. Finally, in Section 8 , we conclude this paper and hint at future research directions.

\section{BACKGROUND}

We present in this section the background information related to view materialization in the cloud. We first introduce a simple fictitious use case that serves as a running example throughout this paper. Then, we describe a typical pricing model in the cloud, illustrated by some of Amazon Web Services (AWS). Then, we briefly recall the principle of view materialization.

\subsection{Running example}

To illustrate our work, we rely on a simulated dataset storing the sales of an international supply chain. Business users need to analyze the total profit per day, month, and year; and per administrative department, region, and country. Table 1 provides an excerpt of this dataset.

Our full dataset stores 10 years (2000-2010) of sale data. Its size is $500 \mathrm{~GB}$. We run over this dataset a query workload $Q$ that includes such queries as $Q_{1}=$ "sales per year and country", whose processing time is 0.2 hour. The size of the $Q$ 's result is $10 \mathrm{~GB}$. A typical materialized view we may consider to optimize overall response time is $V_{1}=$ "sales per month and country", whose processing time is 0.1 hour. The whole set of selected materialized views is denoted $V$. $V$ 's size is $50 \mathrm{~GB}$. Finally, the times to process $Q$ with and without exploiting $V$ are 40 hours and 50 hours, respectively.

\subsection{Cloud pricing policies}

Cloud Service Providers (CSPs) supply a pool of resources, such as hardware (CPU, storage, networks), devel- opment platforms and services. There are many CSPs on the market, such as Amazon, Google and Microsoft. Each CSP offers different services and pricing. This paper relies on a limited, yet representative enough model that includes the main, commonly billed elements, i.e., CPU, storage and bandwidth consumption. In order for the reader to have an overview of such a pricing policy, the following examples present a simplified version of AWS's offer.

In AWS, Elastic Compute Cloud (EC2) [1] provides computing resources. Different instance configurations can be rent (micro, small, large, extra large, etc.) at various prices, as illustrated in Table 2. For example, the cost for a small instance (1.7 GB RAM, 1 EC2 Compute Unit, 160 GB of local storage under Windows) is $\$ 0.12$ per hour. Let us apply this pricing model onto our use case running on two small instances. Without materialized views, processing time is 50 hours, and thus computing cost is $2 \times 0.12 \times 50=\$ 12$. With materialized views, processing time falls down to 40 hours and computing cost down to $2 \times 0.12 \times 40=\$ 9.6$.

\begin{tabular}{|l|l|}
\hline Instance configuration & Price per hour \\
\hline Micro & $\$ 0.03$ \\
\hline Small & $\$ 0.12$ \\
\hline Large & $\$ 0.48$ \\
\hline Extra large & $\$ 0.96$ \\
\hline$\ldots$ & $\ldots$ \\
\hline
\end{tabular}

Table 2: EC2 computing prices

Bandwidth consumption is billed with respect to data volume (Table 3). In this model, input data transfers are free, whereas output data transfer cost varies with respect to data volume, with an earned rate when volume increases. When applying this pricing model onto our use case, the cost of bandwidth consumption (query result of $10 \mathrm{~GB}$ ) is $(10-1) \times 0.12=\$ 1.08$.

\begin{tabular}{|l|l|}
\hline Data volume & Price per month \\
\hline Input data & \\
\hline Any input data & Free \\
\hline Output data & \\
\hline First 1 GB & Free \\
\hline Up to 10 TB & $\$ 0.12$ per GB \\
\hline Next $40 \mathrm{~TB}$ & $\$ 0.09$ per GB \\
\hline Next 100 TB & $\$ 0.07$ per GB \\
\hline$\ldots$ & $\ldots$ \\
\hline
\end{tabular}

Table 3: Amazon bandwidth prices

Finally, the Amazon Simple Storage Service (S3) [2] supplies storage capabilities. In this model, the price varies with respect to data volume, with an earned rate when volume increases (Table 4). In our running example, monthly storage 
price when not using materialized views (500 GB dataset) is $0.14 \times 500=\$ 70$, and $0.14 \times(500+50)=\$ 77$ when using materialized views (additional 50 GB storage).

\begin{tabular}{|l|l|}
\hline Data volume & Price per month \\
\hline First 1 TB & $\$ 0.14$ per GB \\
\hline Next $49 \mathrm{~TB}$ & $\$ 0.125$ per GB \\
\hline Next $450 \mathrm{~TB}$ & $\$ 0.11$ per GB \\
\hline ... & $\ldots$ \\
\hline
\end{tabular}

Table 4: Amazon storage prices

\subsection{Materialized views}

In Database Management Systems, a view is a virtual table associated to a query answer. Views help indirectly save complex queries, present the same data in different forms, support logical independence and reinforce security by masking some pieces of data from unauthorized users. Materializing a view, i.e., storing it physically into a table, further helps improve response time by avoiding to recompute the corresponding query each time the view itself is queried. However, materialized views must be refreshed when source data are updated, which induces some maintenance overhead.

In this work, we assume that we have at our disposal a set of candidate materialized views that have already been generated by an existing materialized view selection method (e.g., [8]). We aim at choosing the best candidates with respect to the cloud's pay-as-you-go model, taking pricing constraints into account before any view materialization.

\section{DATA MANAGEMENT COST MODELS}

This section presents general cost models for data management in the cloud, i.e., without considering the use of materialized views. In cloud computing, customers rent resources to a CSP to run some applications. Figure 1 recalls the costs involved (Section 2.2), i.e., bandwidth consumption for input data transfers and query result retrieval, data storage, and applications' processing time.

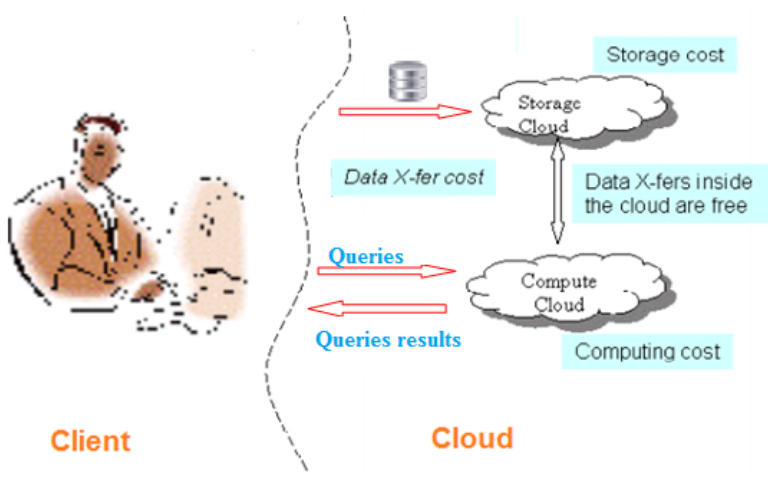

Figure 1: Costs involved in cloud data management

Let $C_{c}$ be the sum of computing costs, $C_{s}$ be the sum of storage costs and $C_{t}$ be the sum of data transfer costs. Then, the total cost $C$ for cloud data management is:

$$
C=C_{c}+C_{s}+C_{t} .
$$

Let us define the general parameters that we use to express our cost models (Table 5), as well as two functions. Function $s()$ returns the size in GB of any of these parameters, e.g. $s(D S)$ is the size of the dataset. Function $t s()$ returns the storage time of any dataset, e.g. $\operatorname{ts}(D S)$ is the storage time of the dataset in the cloud.

\begin{tabular}{|l|l|}
\hline Parameter & Description \\
\hline$D S$ & Dataset \\
\hline$Q=\left\{Q_{i}\right\}_{i=1 . . m}$ & Query workload \\
\hline$R=\left\{R_{i}\right\}_{i=1 . . m}$ & Set of query answers \\
\hline
\end{tabular}

Table 5: General parameters

\subsection{Data transfer cost}

Data transfer cost depends on several parameters: the size of the initial dataset, the amount of data related to queries and query results, the volume of inserted new data and the pricing model applied by the CSP. The total data transfer $\operatorname{cost} C_{t}$ is the product of the CSP's atomic transfer cost $c_{t}$ by the total size of transferred data:

$C_{t}=\left(\sum_{i=1}^{m}\left(s\left(R_{i}\right)+s\left(Q_{i}\right)\right)+(s(D S)+s(\right.$ insertedData $)) \times c_{t}$.

If we adopt Amazon's EC2 like pricing model, which does not charge for input data transfers, we may ignore input queries, the initial data set and inserted data. As a consequence, the total data transfer cost becomes:

$$
C_{t}=\sum_{i=1}^{m} s\left(R_{i}\right) \times c_{t} .
$$

Moreover, recall that Amazon EC2's cost is variable. It is null for the first GB. Then, it becomes $\$ 0.12$ up to $10 \mathrm{~TB}$, and so on (Section 2.2).

Example 1: In our running example, with $10 \mathrm{~GB}$ (with the first one being free) of bandwidth consumption, data transfer cost is: $C_{t}=s\left(R_{Q}\right) \times c_{t}=(10-1) \times 0.12=\$ 1.08$.

\subsection{Computing cost}

Let us pose that queries are executed on computing instances $\left\{I C_{j}\right\}_{j=1 . . n}$. Each instance may bear different performances (with respect to its number of CPUs, its available RAM, etc.), and thus different costs. The cost for renting instance $I C_{j}$ is denoted $c\left(I C_{j}\right)$. It must be paid at each connection to the cloud. Processing time of query $Q_{i}$ on instance $I C_{j}$ is denoted $t_{i j}$. Then, the processing cost of running the set of queries $Q=\left\{Q_{1}, \ldots, Q_{m}\right\}$ is:

$$
C_{c}=\sum_{i=1}^{m} t_{i j} \times c\left(I C_{j}\right) .
$$

Example 2: In our running example, let us consider that the query workload $Q$ is processed on two small instances. Then, its processing cost is: $C_{c}=t \times c(I C)+t \times c(I C)=$ RoundUp $(50) \times 0.12+$ Round $U p(50) \times 0.12=50 \times 0.12+$ $50 \times 0.12=\$ 12$. We must use a function to round processing time up because every started hour is charged.

\subsection{Storage cost}

Storage cost depends on parameters such as the CSP's pricing policy, the size of the data (original dataset and in- 
serted data) and the storage time. We assume that the storage period in the cloud is devided into intervals. In each interval, the size of the stored data is fixed. The total storage cost is the CSP's storage cost function cs(s(DS)) (where DS is the data storage size, that is to say the size of the original data set and the inserted data) multiplied by the sum of sizes of the initial dataset and inserted data multiplied by their respective storage time during the intervals.

$$
C_{s}=\sum_{\text {intervals }} c s(D S) \times\left(t_{\text {end }}-t_{\text {start }}\right) \times s(D S)
$$

Where $t_{\text {start }}, t_{\text {end }}$ are start point and end point of an interval.

Example 3: Using Amazon S3 for storage pricing (referred to table 4) and considering that 0.5 TB (512 GB) data has been stored for 12 months. At the beginning of the eighth month, we insert 2 TB $(2048 \mathrm{~GB})$ of new data in the cloud. Thus we have two intervals. The storage cost is: $C_{s}=\sum_{\text {intervals }} c s(D S) \times\left(t_{\text {end }}-t_{\text {start }}\right) \times s(D S)=(512 \times$ $0,14 \times(7-0)+(512+2048) \times 0,125 \times(12-7)=\$ 2131.76$.

\section{COST MODELS FOR MATERIALIZING VIEWS IN THE CLOUD}

This section presents cost models for materializing views in the cloud, relying on the costs models developed in Section 3. We assume here that queries are executed on a constant number, $n b_{I C}$ of identical instances $I C$. In future work, we shall consider the evaluation process on multiple, variable instances.

Let $V_{\text {cand }}=\left\{V_{k}\right\}_{k=1 . . p}$ be a set of candidate materialized views output by any existing materialized view selection technique.

\subsection{Data transfer cost}

We assume that materialized views are selected on the client's side, outputting final set of materialized views $V$ that are materialized in the cloud. This tactic helps save bandwidth and benefit from the computing performance of the cloud. With materialized views created in the cloud, transfer costs of materialized views are null. As a consequence, total transfers cost $C_{t}$ is not impacted and remains expressed by Formula 3.

\subsection{Computing cost}

Using materialized views implies modifying computing costs, since query processing may exploit materialized views, and views must be materialized and maintained. Computing cost expressed by Formula 4 thus becomes:

$$
C_{c}=C_{\text {processingQ }}+C_{\text {maintenanceV }}+C_{\text {materialization } V}
$$

where $C_{\text {processingQ }}$ is the cost of processing queries, $C_{\text {maintenanceV }}$ is the cost of materialized views' maintenance, and $C_{\text {materializationV }}$ is the cost of materializing views.

\subsubsection{Materialization cost}

If a view is materialized, its associated query must be executed, which must be paid for in the cloud. Let the materialization time of view $V_{k}$ be $t_{\text {materialization }}\left(V_{k}\right)$. We assume that all views are materialized on a constant number of identical instances, $n b_{I C}$.
The total materialization time is:

$$
T_{\text {materializationV }}=\sum_{k=1}^{p} t_{\text {materialization }}\left(V_{k}\right) .
$$

According to Formula 4, materialization cost is:

$$
C_{\text {materializationV }}=T_{\text {materialization } V} \times c(I C) \times n b_{I C} .
$$

Example 4: In our running example with two nodes, we assume that $V=\left\{V_{1}\right\}$, the total materialization time is $T_{\text {materialization }}=\sum_{k=1}^{p} t_{\text {materialization }}\left(V_{k}\right)=$ $t_{\text {materialization }}\left(V_{1}\right)=1$ hour. Then, materialization cost is: $C_{\text {materialisationV }}=t_{\text {materialisation }}\left(V_{1}\right) \times c(I C) \times n b_{I C}=$ $1 \times 0.12 \times 2=\$ 0.24$

\subsubsection{Query processing cost}

When using materialized views, query processing time is defined by two main parameters: query workload $Q$ and set of materialized views $V$. Queries may use the contents of materialized views instead of recomputing their result. Note that we consider that $Q$ is fixed. Since views are usually materialized with respect to a given workload and ours is fixed, then $V$ is also fixed.

Let $t_{i V}$ be the processing time of query $Q_{i}$ when exploiting the set of materialized views $V$.Thus, the total processing time of $Q$ against $V$ is:

$$
T_{\text {processing } Q}=\sum_{i=1}^{m} t_{i V}
$$

Example 5: In our running example, total processing time when using $V$ is 40 hours.

After taking view materialization into account, Formula 4 becomes:

$$
C_{\text {processing } Q}=T_{\text {processing } Q} \times c(I C) \times n b_{I C} .
$$

Example 6: So, the computing cost becomes: $C_{\text {processing } Q}=\sum_{i=1}^{m} t_{i V} \times c(I C) \times n b_{I C}=40 \times 0.12 \times 2=$ $\$ 9.6$.

\subsubsection{Maintenance cost}

The maintenance cost of materialized views is directly proportional to the time required for updating materialized views when they are impacted by modifications of the source dataset. Note that we consider that querying and maintenance do not occur at the same time. For example, queries are posed during day-time and maintenance is performed during night-time. Let the maintenance time of view $V_{k}$ be $t_{\text {maintenance }}\left(V_{k}\right)$. Then, the total maintenance time of $V$ is:

$$
T_{\text {maintenanceV }}=\sum_{k=1}^{p} t_{\text {maintenance }}\left(V_{k}\right) .
$$

Example 7: The maintenance time in the running example is 5 hours.

As a consequence, the cost of maintaining the set of materialized views $V$ is:

$$
C_{\text {maintenanceV }}=T_{\text {maintenanceV }} \times c(I C) \times n b_{I C} .
$$


Example 8: Thus, the maintenance cost is: $C_{\text {maintenanceV }}=T_{\text {maintenanceV }} \times c(I C) \times n b_{I C}=$ $5 \times 0.12 \times 2=\$ 1.2$.

\subsection{Storage cost}

Using materialized views does not impact the storage cost as presented by Formula 5. Exploiting materialized views to enhance query performance implies storing them in the cloud and pay the corresponding cost. As a consequence, some data can be duplicated. In that case DS represents the data set, the duplicated data due to materialized views and possible inserted data. Note that original data and materialized views are stored for the whole considered storage period.

Example 9: In our running example, the data set has been stored for a year, the size of duplicated data due to materialized views is $50 \mathrm{~GB}$. In addition, no data are inserted during the considered period. Thus, we have a single interval and storage cost is $C_{s}=((500+50) \times(12-0) \times 0.14=\$ 924$

\section{OPTIMIZATION PROCESS}

In this section, we investigate how the use of materialized views in the cloud impacts query performance, while trying to minimize overhead storage cost. We propose an algorithm to select the best set of materialized views by exploiting the cost models introduced in Section 4.

\subsection{Objective functions}

Base on the ideas in [20], we distinguish in this section three scenarios, i.e., three objective functions, with respect to the needs and capacity of customers (budget limit, response time limit).

\subsubsection{Budget limit}

Given a predefined financial budget $B l$, our objective in this scenario (denoted $M V_{1}$ ) is to select, from the set of candidate materialized views $V_{\text {cand }}$, the set of views to materialize in the cloud $V$ that minimizes query processing time (Figure 2: The chosen solution are colored in red).

$$
M V_{1}=\left\{\begin{array}{l}
\text { Minimize } T_{\text {processing } Q} \\
C=C_{c}+C_{t}+C_{s} \leq B l
\end{array}\right.
$$

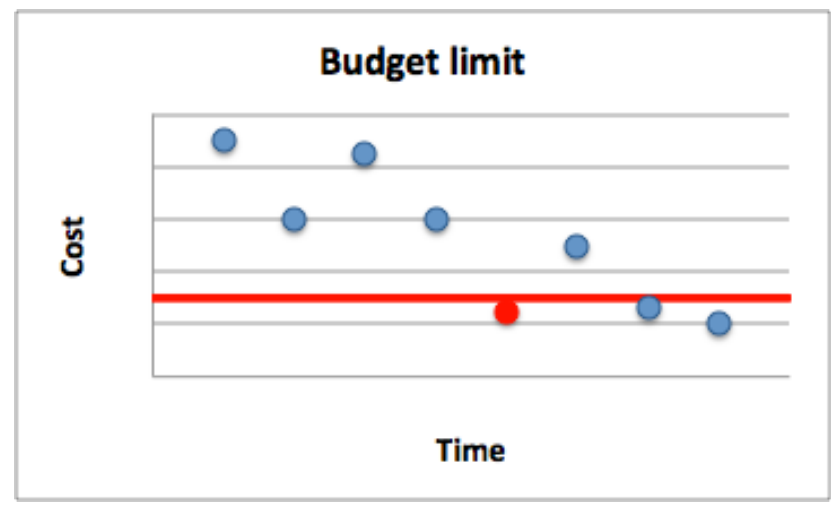

Figure 2: Minimizing processing time under budget constraint

\subsubsection{Response time limit}

Given a predefined response time limit $T l$, our objective in this scenario (denoted $M V_{2}$ ) is to select, from the set of candidate materialized views $V_{\text {cand }}$, the set of views to materialize in the cloud $V$ that minimizes financial cost (Figure 3$)$.

$$
M V_{2}=\left\{\begin{array}{l}
\text { Minimize } C=C_{c}+C_{t}+C_{s} \\
T_{\text {processing } Q} \leq T l
\end{array}\right.
$$

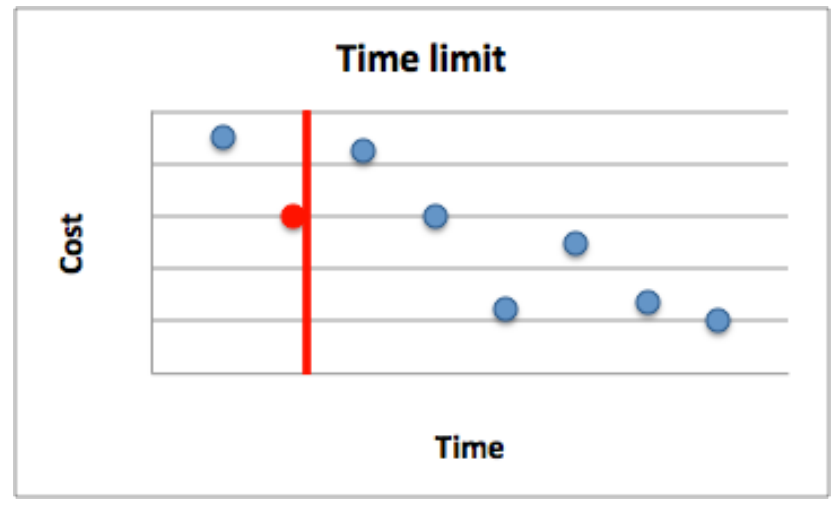

Figure 3: Minimizing cost under response time constraint

\subsubsection{Response time vs. cost tradeoff}

In this scenario (denoted $M V_{3}$ ), our objective is to select, from the set of candidate materialized views $V_{\text {cand }}$, the set of views to materialize in the cloud $V$ that offers the best tradeoff between query processing time and financial cost (Figure 4). To provide the user with control over this process, we introduce a weight parameter on processing time $(\alpha)$ and cost $(1-\alpha)$.

$$
M V_{3}=\text { Minimize }\left(\alpha \times T_{\text {processing } Q}+(1-\alpha) \times C\right)
$$

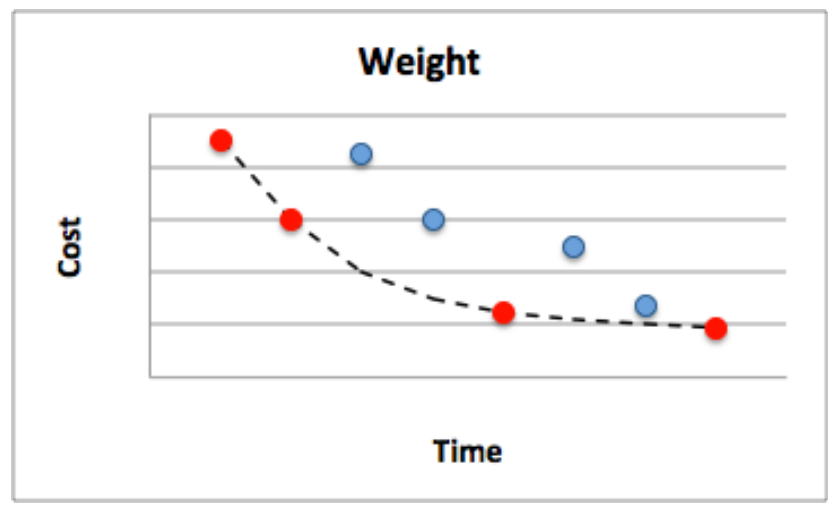

Figure 4: Optimizing response time and cost

\subsection{Optimization algorithm}

In order to select the set of views to materialize, we rely on an existing algorithm such as [8] enabling to obtain a set of candidate views $V_{\text {cand }}$. Then we solve the Knapsack 0/1 
problem [14] considering this set. To solve this problem, we have opted for a dynamic programming approach.

As a input, we provide elements of our cost models. For example, in the case of MV1 (budget limit), the result is: $V=K$ napsack $\left(V_{\text {cand }}, F_{C t}, F_{C c}, F_{C s}, B l\right)$, where $F_{C t}, F_{C c}$, $F_{C s}$ are functions which represent the parameters of the models described in the section 4 . In particular, $F_{C t}$ involves transfer cost parameters (section 4.1 ), $F_{C c}$ involves computing cost parameters(section 4.2), and $F_{C s}$ involves storage cost parameters (section 4.3).

\section{EXPERIMENTAL VALIDATION}

In this section, we describe the overall setup of our preliminary experimentation effort and the results we have obtained from it. This experiment is realized on both sides: in the cloud and on the client's side. The dataset and materialized views are stored in the cloud, and queries are processed in the cloud. The view selection algorithms are implemented in Java on the client's side. The idea is to select views on the client's side and to materialize them in the cloud.

\subsection{Experimental setup}

The experiments were conducted on a virtual cluster composed of five virtual machines with a 50 GB disk, 2 GB of RAM and 4 vCPU. This virtual cluster is deployed on a physical architecture consisting of two bi-pros $493 \mathrm{GHz}$ with $32 \mathrm{~GB}$ of RAM, interconnected by a $1 \mathrm{~GB} / \mathrm{s}$ network in a $8 \times 2$ DRS cluster; and a bi-pro $2,526 \mathrm{GHz}$ with hyperthreading enabled, outside of the DRS cluster, with 16 logical cores with $72 \mathrm{~GB}$ of RAM on a $1 \mathrm{~GB} / \mathrm{s}$ network. All machines feature Hadoop (version 0.20.2) [3] and Pig Latin (version 0.7.0) [23]. Queries are written in Pig Latin and are executed by the Pig compiler on the Hadoop cluster within the MapReduce framework [17]. On the client's side, algorithms are implemented on a PC Dual Core 2 T6660 2.2GHz, with 2 GB of RAM.

We used in our experiments a subset of the dataset from our case study (Section 2.1), whose size is 10 GB. The workload we applied on the dataset was made of 10 queries that calculate the total profit per day, month, year and per country, department, and region such as "per year and per country".

\subsection{Experimental results}

We experimented the three scenarios $M V_{1}, M V_{2}$ and $M V_{3}$ described in Section 5.1, with a variable workload made of 3 , 5, and 10 queries, with and without exploiting materialized views. Our results are plotted on Figure 5. They clearly show that, in all scenarios, creating materialized views in the cloud is desirable.

In scenario $M V_{1}$, a significantly better response time is achieved with materialized views, under the same budget constraint than without (Figure 5(a)). The improved performance rates are shown in the Table 6 .

\begin{tabular}{|l|l|l|}
\hline Number of queries & Budget limit & IP Rate \\
\hline 3 & $\$ 0.8$ & $25 \%$ \\
\hline 5 & $\$ 1.2$ & $36 \%$ \\
\hline 10 & $\$ 2.4$ & $60 \%$ \\
\hline
\end{tabular}

Table 6: Improved performance rates between with and without materialized views under the same budget limit in the scenario $M V_{1}$
In scenario $M V_{2}$, a significantly lower global cost is achieved with materialized views, under the processing time constraint than without (Figure 5(b)). The improved cost rates are shown in the Table 7 .

\begin{tabular}{|l|l|l|}
\hline Number of queries & Time limit & IC Rate \\
\hline 3 & $0.57 \mathrm{~h}$ & $75 \%$ \\
\hline 5 & $0.99 \mathrm{~h}$ & $72 \%$ \\
\hline 10 & $2.24 \mathrm{~h}$ & $75 \%$ \\
\hline
\end{tabular}

Table 7: Improved cost rates between with and without materialized views under the same time limit in the scenario $M V_{2}$

Similarly, in scenario $M V_{3}$, materialized views help achieve a tradeoff (chosen by the client) between cost and response time, whether the priority is put on cost (Figure 5(c)) or response time (Figure $5(\mathrm{~d})$ ). Improved tradeoff rates are shown in the Table 8 .

\begin{tabular}{|l|l|l|}
\hline Number of queries & Rate $(\alpha=0.3)$ & Rate $(\alpha=0.7)$ \\
\hline 3 & $55 \%$ & $32 \%$ \\
\hline 5 & $50 \%$ & $35 \%$ \\
\hline 10 & $68 \%$ & $45 \%$ \\
\hline
\end{tabular}

Table 8: Improved tradeoff rates between with and without materialized views in the scenario $M V_{3}$

\section{RELATED WORK}

Although there has been a lot of research related to the cloud for a couple of years, relatively few approaches relate to the optimization of data management in the cloud. In this section, we provide a brief overview of cost models, databaselike features and the use of optimization techniques in the cloud.

Cost models have been proposed in the cloud for scheduling of dataflows with regard to monetary cost and/or completion time [20], and cost amortization of data structures to ensure the economic viability of the provider [19], particularly for self-tuned caching [16]. Other cost models have been developed for a real-life astronomy application using the Amazon cloud fee structure [9, 18]. The cost performance tradeoffs of different execution and resource provisioning plans have been simulated, showing that by provisioning the right amount of storage and computing resources, cost can be significantly reduced with no significant impact on application performance [18]. The performance of three workflow applications with different I/O, memory and $\mathrm{CPU}$ requirements has also been compared on Amazon EC2 and a typical high-performance cluster (HPC) to identify what applications achieve the best performance in the cloud at the lowest cost [9].

Recent research takes interest in various aspects of database and decision support technologies in the cloud. For example, different studies investigate the storage and processing of structured data [13], the optimization of join queries, and how to support analysis operations such as aggregation [15]. Cloud data warehousing and OLAP systems also raise various problems related to storage and query performance [22]. Adaptations of these technologies to the cloud's specificities are also addressed, e.g., pay-as-you-go pricing and elasticity [7], or the calculation of OLAP cuboids using the MapReduce runtime environment [25]. 


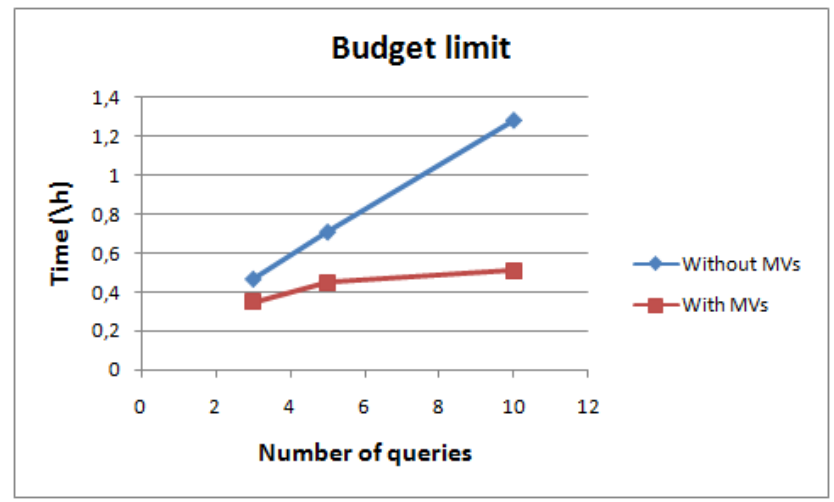

(a) Scenario $M V_{1}$ (budget limit)

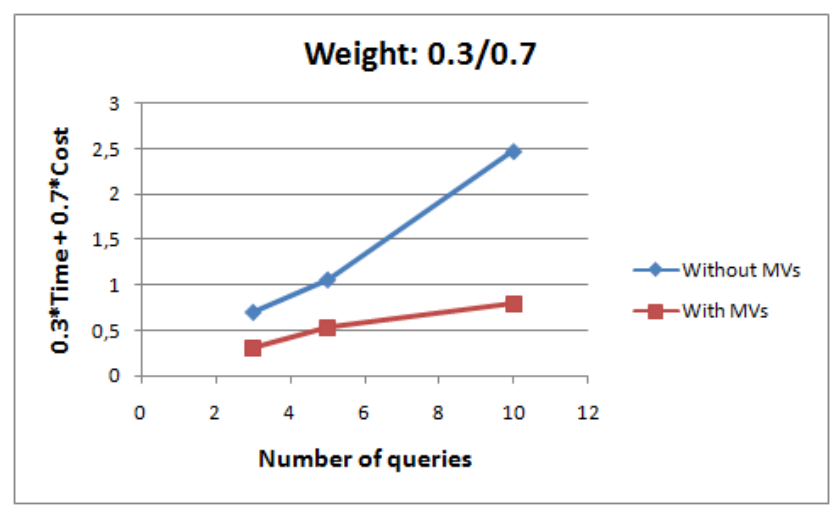

(c) Scenario $M V_{3}$ (tradeoff) with $\alpha=0.3$

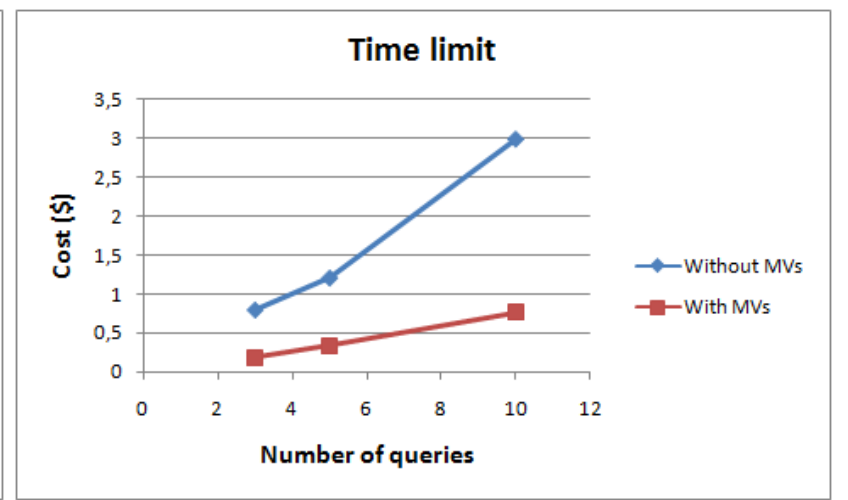

(b) Scenario $M V_{2}$ (response time limit)

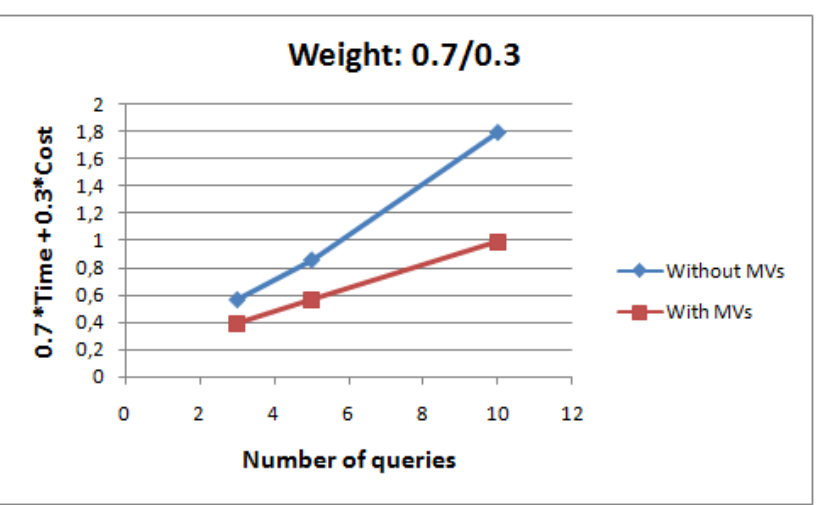

(d) Scenario $M V_{3}$ (tradeoff) with $\alpha=0.65$

Figure 5: Experimental results of application of materialized views

Finally, although massive scale distributed database systems such as BigTable have begun to incorporate indexes and materialized views [11], the many techniques developed for maintaining materialized views in traditional, relational databases [5], such as incremental view maintenance [12], deferred maintenance [27] and distributed view maintenance [21], have not been incorporated into the cloud yet. This is thus the main incentive for our own approach.

\section{CONCLUSION}

In this paper, we proposed an approach for decreasing the cost of data management in the cloud, by using a classical database performance optimization technique, i.e., view materialization. Our main contributions are novel cost models that complement the existing materialized view cost models with a monetary cost component that is primordial in the cloud. Then, we exploit these cost models into an optimization process that achieves a tradeoff between computing power enhancement and view materialization, under budget constraints. Our first experimental validation hints that view materialization is always desirable.

Many perspectives are opened by this preliminary research. We indeed have many plans on improving our cost models. First, we should include pricing models from several CSPs but Amazon, to render our cost models more versatile. Second, it is well-known that optimization techniques are the most efficient when combined, e.g., when materialized views and index are jointly exploited [6]. Thus, we should incorporate indexing, caching and/or fragmentation into our approach. Third, we should exploit the specificities of cloud languages (such as HiveQL [26], Jaql [10] and Pig Latin [23]) with respect to semantics and parallelism to further improve performance. Finally, in this paper, we worked on one single cloud instance, thus minimizing the primordial elasticity characteristic of the cloud. Thus, we should expand our cost models on variable resources.

Another line of future work relates to the experimental validation of our proposals. Here, due to limited resources, we experimented our cost models on a small cluster and a toy dataset. Thus, we plan to design a wider-scale experimentation plan that would exploit a full-fledged database or data warehouse benchmark, such as TPC-E [4] or the Star Schema Benchmark [24], onto AWS or other infrastructure providers.

\section{Acknowledgment}

This work is sponsored by the ANR under grant SYSEO ANR-10-TECSAN-005-01. We would like to sincerely thank all the colleagues in IRSTEA, LIMOS and ERIC laboratories for the interesting discussions and the reviewers for their extremely insightful remarks for improving this paper.

\section{REFERENCES}

[1] Amazon ec2. http://aws.amazon.com/ec2/, February 2012.

[2] Amazon s3. http://aws.amazon.com/s3/, February 2012. 
[3] Hadoop. http://hadoop.apache.org/, February 2012.

[4] Tpc-e. http://www.tpc.org/tpce, February 2012.

[5] P. Agrawal, A. Silberstein, B. F. Cooper, U. Srivastava, and R. Ramakrishnan. Asynchronous view maintenance for vlsd databases. In Proceedings of the ACM SIGMOD International Conference on Management of Data, pages 179-192, Providence, USA, 2009

[6] K. Aouiche and J. Darmont. Data mining-based materialized view and index selection in data warehouses. Journal of Intelligent Information Systems, 33(1):65-93, 2009.

[7] M. Armbrust, A. Fox, R. Griffith, A. D. Joseph, R. H. Katz, A. Konwinski, G. Lee, D. A. Patterson, A. Rabkin, I. Stoica, and M. Zaharia. A view of cloud computing. Communications of the ACM, 53(4):50-58, 2010.

[8] X. Baril and Z. Bellahsene. Selection of materialized views: A cost-based approach. In International Conference on Advanced Information Systems Engineering, pages 665-680, Klagenfurt, Austria, 2003.

[9] G. B. Berriman, E. Deelman, G. Juve, M. Regelson, and P. Plavchan. The application of cloud computing to astronomy: A study of cost and performance. CoRR, abs/1010.4813, 2010.

[10] K. S. Beyer, V. Ercegovac, R. Gemulla, A. Balmin, M. Y. Eltabakh, C.-C. Kanne, F. Özcan, and E. J. Shekita. Jaql: A scripting language for large scale semistructured data analysis. PVLDB, 4(12):1272-1283, 2011

[11] M. J. Cafarella, E. Y. Chang, A. Fikes, A. Y. Halevy, W. C. Hsieh, A. Lerner, J. Madhavan, and S. Muthukrishnan. Data management projects at google. SIGMOD Record, 37(1):34-38, 2008.

[12] S. Ceri and J. Widom. Deriving production rules for incremental view maintenance. In Proceedings of the International Conference on Very Large Data Bases, September, pages 577-589, Barcelona, Spain, 1991.

[13] D. Chatziantoniou and E. Tzortzakakis. Asset queries: a declarative alternative to mapreduce. SIGMOD Record, 38(2):35-41, 2009.

[14] V. Chvatal. Hard knapsack problems. Operations Research, 28:1402-1411, 1980.

[15] T. Condie, N. Conway, P. Alvaro, J. M. Hellerstein, J. Gerth, J. Talbot, K. Elmeleegy, and R. Sears. Online aggregation and continuous query support in mapreduce. In Proceedings of the ACM SIGMOD International Conference on Management of Data, pages 1115-1118, Indianapolis, USA, 2010.

[16] D. Dash, V. Kantere, and A. Ailamaki. An economic model for self-tuned cloud caching. In Proceedings of the International Conference on Data Engineering, pages 1687-1693, Shanghai, China, 2009.

[17] J. Dean and S. Ghemawat. Mapreduce: simplified data processing on large clusters. Communications of the ACM, 51(1):107-113, 2008.

[18] E. Deelman, G. Singh, M. Livny, G. B. Berriman, and J. Good. The cost of doing science on the cloud: the montage example. In Proceedings of the ACM/IEEE Conference on High Performance Computing, page 50, Austin, USA, 2008.
[19] V. Kantere, D. Dash, G. Gratsias, and A. Ailamaki. Predicting cost amortization for query services. In Proceedings of the ACM SIGMOD International Conference on Management of Data, pages 325-336, Athens, Greece, 2011.

[20] H. Kllapi, E. Sitaridi, M. M. Tsangaris, and Y. E. Ioannidis. Schedule optimization for data processing flows on the cloud. In Proceedings of the ACM SIGMOD International Conference on Management of Data, pages 289-300, Athens, Greece, 2011.

[21] G. Luo, J. F. Naughton, C. J. Ellmann, and M. Watzke. A comparison of three methods for join view maintenance in parallel rdbms. In Proceedings of the International Conference on Data Engineering, pages 177-188, Bangalore, India, 2003.

[22] H. Mahboubi and J. Darmont. Enhancing xml data warehouse query performance by fragmentation. In Proceedings of the ACM Symposium on Applied Computing, pages 1555-1562, Honolulu, USA, 2009.

[23] C. Olston, B. Reed, U. Srivastava, R. Kumar, and A. Tomkins. Pig latin: a not-so-foreign language for data processing. In Proceedings of the ACM SIGMOD International Conference on Management of Data, pages 1099-1110, Vancouver, BC, Canada, 2008.

[24] P. E. O'Neil, E. J. O'Neil, X. Chen, and S. Revilak. The star schema benchmark and augmented fact table indexing. In Proceedings of the TPC Technology Conference on Performance Evaluation and Benchmarking, pages 237-252, Lyon, France, 2009.

[25] K. Sergey and K. Yury. Applying map-reduce paradigm for parallel closed cube computation. In Proceedings of the International Conference on Advances in Databases, Knowledge, and Data Applications, pages 62-67, Gosier, France, 2009.

[26] A. Thusoo, J. S. Sarma, N. Jain, Z. Shao, P. Chakka, S. Anthony, H. Liu, P. Wyckoff, and R. Murthy. Hive a petabyte scale data warehouse using hadoop. In Proceedings of the International Conference on Data Engineering, pages 996-1005, Long Beach, USA, 2010.

[27] J. Zhou, P.-A. Larson, and H. G. Elmongui. Lazy maintenance of materialized views. In Proceedings of the International Conference on Very Large Data Bases, pages 231-242, Vienna, Austria, 2007. 